\title{
Nuclear Magnetic Resonance
}

National Cancer Institute

\section{Source}

National Cancer Institute. Nuclear Magnetic Resonance. NCI Thesaurus. Code C16921.

A physical phenomenon involving the interaction of atomic nuclei placed in an external magnetic field with an applied electromagnetic field oscillating at a particular frequency. Magnetic conditions within the material are measured by monitoring the radiation absorbed and emitted by the atomic nuclei. It is the underlying principle of Magnetic Resonance Imaging (MRI). 